\title{
CONTROL FOR SCHRÖDINGER OPERATORS ON TORI
}

\author{
Nicolas Burq and Maciej Zworski
}

\begin{abstract}
A well-known result of Jaffard states that an arbitrary region on a torus controls, in the $L^{2}$ sense, solutions of the free stationary and dynamical Schrödinger equations. In this paper, we show that the same result is valid in the presence of a potential, that is for Schrödinger operators, $-\Delta+V, V \in \mathcal{C}^{\infty}$.
\end{abstract}

\section{Introduction}

We show how simple methods introduced in $[9,10,21]$ (see also $[15,20]$ ) for the study of the equation

$$
(-\Delta-\lambda) u(z)=f(z), \quad z \in \mathbb{T}^{2}:=\mathbb{R}^{2} / A \mathbb{Z} \times B \mathbb{Z}, \quad A, B \in \mathbb{R} \backslash\{0\},
$$

where $\lambda \rightarrow \infty$, and the control of

$$
\mathrm{i} \partial_{t} u(t, z)=-\Delta u(t, z), \quad z \in \mathbb{T}^{2} .
$$

can be adapted to obtain similar results for the equations

$$
(-\Delta+V(z)-\lambda) u(z)=f(z), \quad z \in \mathbb{T}^{2}
$$

and

$$
\mathrm{i} \partial_{t} u(t, z)=(-\Delta+V(z)) u(t, z), \quad z \in \mathbb{T}^{2},
$$

where $V \in \mathcal{C}^{\infty}\left(\mathbb{T}^{2}\right)$ is a smooth real valued potential.

The first theorem concerns solutions of the stationary Schrödinger equation and is applicable to high-energy eigenfunctions:

Theorem 1.1. Let $\Omega \subset \mathbb{T}^{2}$ be a non-empty open set. There exists a constant $K=$ $K(\Omega)$, depending only on $\Omega$, such that for any solution of (1.1) we have

$$
\|u\|_{L^{2}\left(\mathbb{T}^{2}\right)} \leq K\left(\|f\|_{L^{2}\left(\mathbb{T}^{2}\right)}+\|u\|_{L^{2}(\Omega)}\right) .
$$

This means that $u$ on $\mathbb{T}^{2}$ is controlled by $u$ in $\Omega$, in the $L^{2}$ sense. The next result, which is in fact more general, concerns the dynamical Schrödinger equation:

Theorem 1.2. Let $\Omega \subset \mathbb{T}^{2}$ be any (non-empty) open set and let $T>0$. There exists a constant $K=K(\Omega, T)$, depending only on $\Omega$ and $T$, such that for any solution of (1.2) we have

$$
\|u(0, \bullet)\|_{L^{2}\left(\mathbb{T}^{2}\right)}^{2} \leq K \int_{0}^{T}\|u(t, \bullet)\|_{L^{2}(\Omega)}^{2} d t .
$$

Received by the editors July 8, 2011. 
An estimate of this type is called an observability result. Once we have it, the HUM method (see [19]) automatically provides the following control result:

Theorem 1.3. Let $\Omega \subset \mathbb{T}^{2}$ be any (non empty) open set and let $T>0$. For any $u_{0} \in L^{2}\left(\mathbb{T}^{2}\right)$, there exists $f \in L^{2}((0, T) \times \Omega)$ such that the solution of the equation

$$
\left(i \partial_{t}+\Delta-V(z)\right) u(t, z)=f \mathbb{1}_{(0, T) \times \Omega}(t, z), \quad u(0, \bullet)=u_{0},
$$

satisfies

$$
u(T, \bullet) \equiv 0 .
$$

By applying Theorem 1.2 to the initial data $u(0, \bullet)=u$, it is easy to see that Theorem 1.1 follows from Theorem 1.2 and the Duhamel formula. As a consequence, we will restrict our attention to Theorem 1.2.

We remark that Theorem 1.1 implies Theorem 1.2 for some large $T$ - see [8, 22]. If Theorem 1.1 held with $\|f\|_{L^{2}}$ on the right-hand side replaced by $\|f\|_{H^{-\epsilon}}$, for any $\epsilon>0$, then it would imply Theorem 1.2 - see [8, Theorem 4]. In particular, this holds when the geometric control condition of Bardos-Lebeau-Rauch [2] and Lebeau $[18]$ is satisfied (see also $[6,7,13]$ ). We stress that the $\epsilon$ improvement of regularity in Theorem 1.1 is not possible.

In the case of $V \equiv 0$, the estimates (1.3) and (1.4) were proved by Jaffard [16] and Haraux [14] using Kahane's work [17] on lacunary Fourier series.

For a presentation of control theory for the Schrödinger equation, we refer to $[18]$ — see also [4, 23] and [8, Section 3].

We conclude this introduction with comments about a natural class of potentials for which the theorems above should hold. When $V \in L^{\infty}$ and $\|V\|_{L^{\infty}} \ll 1$ a perturbation argument shows that (1.3) and (1.4) follow from results with $V=0$.

The methods of this paper can be extended to the case of $V \in C^{0}\left(\mathbb{T}^{2}\right)$ by first showing that the constant in the high-frequency estimate (3.1) is independent of $V$ for $V$ in a bounded subset of $L^{\infty}$ and then using approximation and a perturbation argument. The restriction that $V$ is real is not essential but makes the writing easier as we can use the calculus of self-adjoint operators.

Conjecture 1.1. Theorems 1.1-1.3 hold for $V \in L^{\infty}\left(\mathbb{T}^{2} ; \mathbb{C}\right)$. Theorems 1.2 and 1.3 hold for time-dependent potentials $V(t, z) \in L^{\infty}\left(\mathbb{R} \times \mathbb{T}^{2} ; \mathbb{C}\right)$.

\section{Preliminaries}

In this section, we will recall the basic control result $[3,9]$ for rectangles, and the normal form theorem based on Moser-averaging method [24].

The following result [3] is related to some earlier control results of Haraux [14] and Jaffard $[16]^{1}$ :

Proposition 2.1. Let $\Delta$ be the Dirichlet, Neumann, or periodic Laplace operator on the rectangle $R=[0, a]_{x} \times[0, b]_{y}$. Then for any open non-empty $\omega \subset R$ of the form $\omega=\omega_{x} \times[0, a]_{y}$, there exists $C$ such that for any solutions of

$$
(\Delta-z) u=f \quad \text { on } R, \quad u \uparrow_{\partial R=0,}
$$

\footnotetext{
${ }^{1}$ We remark that as noted in [3] the result holds for any product manifold $M=M_{x} \times M_{y}$, and the proof is essentially the same.
} 
we have

$$
\|u\|_{L^{2}(R)}^{2} \leq C\left(\|f\|_{L^{2}\left([0, b]_{y}\right) ; H^{-1}\left([0, a]_{x}\right)}^{2}+\|u\|_{L^{2}(\omega)}^{2}\right) .
$$

Proof. We will consider the Dirichlet case (the proof is the same in the other two cases) and decompose $u, f$ in terms of the basis of $L^{2}([0, b])$ formed by the Dirichlet eigenfunctions $e_{k}(y)=\sqrt{2 / b} \sin (2 k \pi y / b)$,

$$
u(x, y)=\sum_{k} e_{k}(y) u_{k}(x), \quad f(x, y)=\sum_{k} e_{k}(y) f_{k}(x),
$$

we get for $u_{k}, f_{k}$ the equation

$$
\left(-\Delta_{x}-\left(z-(2 k \pi / b)^{2}\right)\right) u_{k}=f_{k}, \quad u_{k}(0)=u_{k}(1)=0 .
$$

We now claim that

$$
\left\|u_{k}\right\|_{L^{2}\left([0,1]_{x}\right)}^{2} \leq C\left(\left\|f_{k}\right\|_{H^{-1}\left([0,1]_{x}\right)}^{2}+\left\|u_{k} \uparrow \omega_{x}\right\|_{L^{2}(\omega)}^{2}\right),
$$

from which, by summing the squares in $k$, we get (2.2).

To see (2.5) we can use the propagation result below in dimension one, but in this case an elementary calculation is easily available — see [9].

The next proposition is the dynamical version of Proposition 2.1, which will be crucial in the proof of Theorem 1.2. However, we change the assumptions on $u$.

Proposition 2.2. Let $R=[0, a]_{x} \times[0, b]_{y}$, and let $\omega=\omega_{x} \times[0, b]$, where $\omega_{x}$ is an open subset of $[0, a]$. Suppose that for $W \in \mathcal{C}^{\infty}(\mathbb{R}), W(x+a)=W(x)$,

$$
\mathrm{i} \partial_{t} u(t, x, y)=(-\Delta+W(x)) u(t, x, y) \text { on } \mathbb{R} \times \mathbb{R}^{2},
$$

and that, for some $\gamma \in \mathbb{R}$, $u$ satisfies the following periodicity condition:

$$
u(t, x+k a, y+\ell b)=u(t, x, y+k \gamma), \quad k, \ell \in \mathbb{Z} .
$$

Then for all $T>0$ there exists $K>0$ such that

$$
\|u(0, \bullet)\|_{L^{2}(R)}^{2} \leq K \int_{0}^{T}\|u(t, \bullet)\|_{L^{2}(\omega)}^{2} d t .
$$

Remark 2.1. Unitarity of the propagator $\exp (-\mathrm{i} t(-\Delta+W))$ shows that the $(0, T)$ range integration on the right hand side of (2.6) can be replaced by $\left(T^{\prime}, T\right)$ for any $0 \leq T^{\prime}<T$. Same statement is true in the case of (1.4).

Proof. As in the proof of Proposition 2.1 we reduce the estimate to an estimate in one dimension.

To do that we see that (2.7) implies that $u$ is periodic in $y$ and hence can be expanded into a Fourier series:

$$
\begin{aligned}
& u(t, x, y)=\sum_{n \in \mathbb{Z}} \mathrm{e}^{-\mathrm{i} t(n \pi / b)^{2}} u_{n}(t, x) \mathrm{e}^{2 \pi \mathrm{i} n y / b} \\
& u_{n}(t, x):=\mathrm{e}^{\mathrm{i} t(n \pi / b)^{2}} \frac{1}{b} \int_{0}^{b} u(t, x, y) \mathrm{e}^{-2 \pi \mathrm{i} n y / b} d y .
\end{aligned}
$$


The condition (2.7) now means that

$$
\begin{aligned}
u_{n}(t, x+a) & =\mathrm{e}^{2 \pi \mathrm{i} \gamma n / b} u_{n}(t, x)=\mathrm{e}^{2 \pi \mathrm{i} \gamma_{n}} u_{n}(t, x), \\
\gamma_{n} & =\gamma n / b-[\gamma n / b], \quad 0 \leq \gamma_{n}<1,
\end{aligned}
$$

that is, the periodicity in $x$ is replaced by a Floquet periodicity condition.

Proposition 2.2 then follows from Lemma 2.1 below.

Lemma 2.1. Let $\omega_{x} \subset[0, a]$ be any open set. Suppose that $v \in L_{l o c}^{2}(\mathbb{R} \times[0, a])$ solves

$$
\left(\mathrm{i} \partial_{t}-D_{x}^{2}-W(x)\right) v=0, \quad W(x+a)=W(x)
$$

and for some $\alpha, 0 \leq \alpha<1, v$ satisfies a Floquet periodicity condition,

$$
v(t, x+a)=\mathrm{e}^{2 \pi \mathrm{i} \alpha} v(x) .
$$

Then for any $T$ there exists $C$, independent of $\alpha$, such that

$$
\|v(0, \bullet)\|_{L^{2}([0, a])}^{2} \leq C \int_{0}^{T}\|v(t, \bullet)\|_{L^{2}\left(\omega_{x}\right)}^{2} d t .
$$

Proof. We use the semi-classical approach developed by Lebeau [18, Theorem 3.1] although the situation is simpler here as we are dealing with internal controls in dimension 1.

Writing $w(x):=\mathrm{e}^{-2 \pi \mathrm{i} \alpha x / a} v(x)$, we obtain a periodic function $w$ satisfying

$$
\left(\mathrm{i} \partial_{t}-\left(D_{x}+\beta\right)^{2}-W(x)\right) w=0, \quad \beta:=\frac{2 \pi \alpha}{a} .
$$

The argument from [18], (used in Sections 3 and 4, below — see Remark 3.1 and also [5]) applies and shows uniformity in $\alpha$. For reader's convenience we provide more details in the appendix.

Next, we present a slight variation of the well-known normal form result — see [24] where it was used in the case of Zoll manifolds (of which the circle is a trivial example). Our version can also be seen as a special case of the normal form in [11]

We start by introducing some notation: we have the spaces of standard pseudodifferential operators $\Psi^{m}(\mathbb{T}), \Psi^{m}\left(\mathbb{T}^{2}\right)$, while

$$
\mathcal{C}^{\infty} \otimes \Psi^{m}:=\mathcal{C}^{\infty}\left(\mathbb{T}_{x}^{1}\right) \otimes \Psi^{m}\left(\mathbb{T}_{y}\right),
$$

denotes the space of semiclassical pseudo-differential operators (of order m) in $y$, depending smoothly on $x$ as a parameter.

To makes things transparent we first present normal form results for tori.

Proposition 2.3. Let $\chi \in \mathcal{C}_{\mathrm{c}}^{\infty}\left(\mathbb{R}^{2}\right)$ be equal to 0 in a neighbourhood of $\eta=0$. Suppose that $V(x, y) \in \mathcal{C}^{\infty}\left(\mathbb{T}^{1} \times \mathbb{T}^{1}\right)$. Then there exist operators

$$
Q\left(x, y, h D_{y}\right) \in \mathcal{C}^{\infty} \otimes \Psi^{0}, \quad R\left(x, y, h D_{x}, h D_{y}\right) \in \Psi^{0}\left(\mathbb{T}^{2}\right),
$$

such that

$$
\begin{aligned}
(I & +h Q)\left(D_{y}^{2}+V(x, y)\right) \chi\left(h D_{x}, h D_{y}\right) \\
& =\left(D_{y}^{2}+V_{0}(x)\right)(I+h Q) \chi\left(h D_{x}, h D_{y}\right)+h R
\end{aligned}
$$


where

$$
V_{0}(x)=\frac{1}{2 \pi} \int_{\mathbb{T}^{1}} V(x, y) d y
$$

Proof. Indeed, we have

$$
\begin{aligned}
(I+ & h Q)\left(D_{y}^{2}+V(x, y)\right) \chi\left(h D_{x}, h D_{y}\right) \\
& -\left(D_{y}^{2}+V_{0}(x)\right)(\mathrm{Id}+h Q) \chi\left(h D_{x}, h D_{y}\right) \\
= & \left(h\left[Q, D_{y}^{2}\right]+V(x, y)-V_{0}(x)+h R_{1}\right) \chi\left(h D_{x}, h D_{y}\right)
\end{aligned}
$$

with $R_{1} \in \mathcal{C}^{\infty} \otimes \Psi^{0}$. The pseudo-differential calculus shows that to obtain (2.12), it is enough to find $q \in \mathcal{C}_{\mathrm{c}}^{\infty}\left(\mathbb{T}^{2} \times \mathbb{R}\right)$ such that

$$
\left(-\frac{2}{i} \eta \partial_{y} q(x, y, \eta)+V(x, y)-V_{0}(x)\right) \chi(\xi, \eta)=0 .
$$

Since $\chi$ vanishes near $\eta=0$, we can find $\zeta \in \mathcal{C}_{\mathrm{c}}^{\infty}(\mathbb{R} \backslash\{0\})$ equal to 1 on the support of $\chi$, and we can solve (2.14) by taking

$$
q(x, y, \eta)=\frac{\mathrm{i} \zeta(\eta)}{2 \eta} \int_{0}^{y}\left(V\left(x, y^{\prime}\right)-V_{0}(x)\right) d y^{\prime} .
$$

We note that by construction, $V_{0}(x)-V(x, y)$ has $y$-mean equal to 0 and consequently the function $q$ defined in $(2.15)$ is periodic.

Corollary 2.1. There exists operators

$$
W=W\left(x, y, h D_{x}, h D_{y}\right) \in \Psi^{0}\left(\mathbb{T}^{2}\right), \quad R=R\left(x, y, h D_{x}, h D_{y}\right) \in \Psi^{0}\left(\mathbb{T}^{2}\right),
$$

such that

$$
\begin{gathered}
(I+h Q)\left(D_{x}^{2}+D_{y}^{2}+V(x, y)\right) \chi\left(h D_{x}, h D_{y}\right) \\
=\left(\left(D_{x}^{2}+D_{y}^{2}+V_{0}(x)\right)(I+h Q)+W\right) \chi\left(h D_{x}, h D_{y}\right)+h R, \\
W(x, y, 0, \eta) \equiv 0 .
\end{gathered}
$$

Proof. Indeed, the same calculation as above shows that by symbolic calculus, we can take

$$
W(x, y, \xi, \eta)=\frac{2}{i} \xi \partial_{x} q(x, y, \eta) \widetilde{\chi}(\xi, \eta),
$$

where $\tilde{\chi} \in \mathcal{C}_{\mathrm{c}}^{\infty}\left(\mathbb{R}^{2}\right)$ is equal to one on the support of $\chi$.

In the case of irrational tori $\mathbb{T}^{2} \simeq[0, A] \times[0, B], A / B \notin \mathbb{Q}$, we need slightly more complicated versions of Proposition 2.3 and Corollary 2.1. They involve covering $\mathbb{T}^{2}$ by a strip.

Let us consider a constant rational vector field on the torus given by a direction

$$
\Xi_{0}=c(n A, m B), \quad n, m \in \mathbb{Z}, \quad c \in \mathbb{R} \backslash\{0\} .
$$

In our argument below the directions corresponding to phase-space concentration of the solution to (1.2) will be necessarily rational and $\Xi_{0}$ will be chosen to be an isolated direction of that type.

As shown in Figure 1, we can find a strip bounded in the direction of $\Xi_{0}$ and covering $\mathbb{T}^{2}$. If the torus is itself rational (that is $A / B \in \mathbb{Q}$ in $(3.4)$ ), we can find a rectangle $R$ with sides parallel to $\Xi_{0}$ and $\Xi_{0}^{\perp}$, which covers $\mathbb{T}^{2}$. 

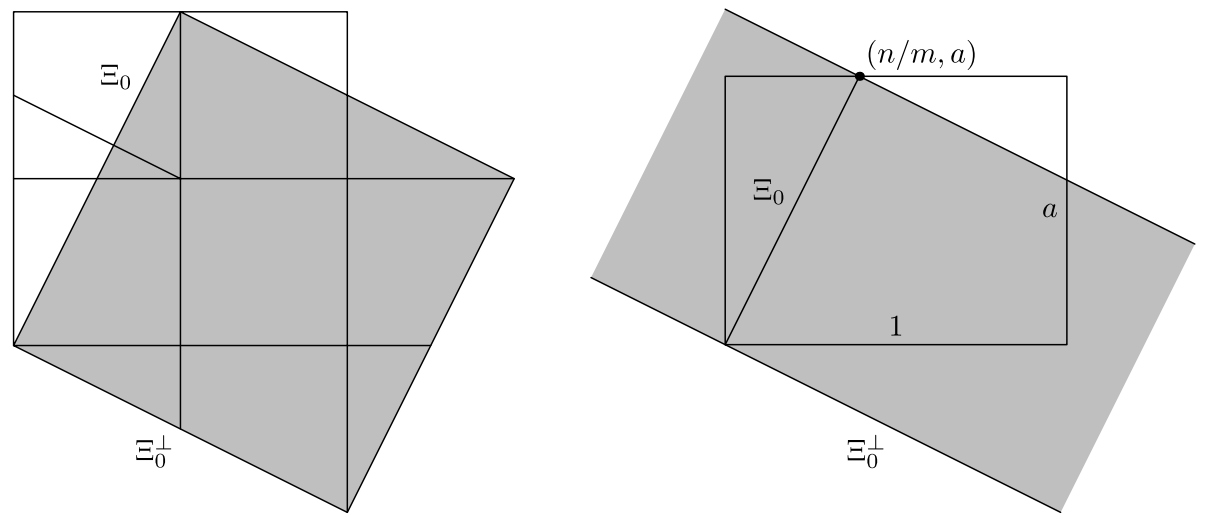

Figure 1. On the left, a rectangle, $R$, covering a rational torus $\mathbb{T}^{2}$. In that case, we obtain a periodic solution on $R$. On the right, the irrational case: the strip with sides $m \Xi_{0} \times \mathbb{R}_{0}^{\perp}, \Xi_{0}=(n / m, a)$ (not normalized to have norm one), also covers the torus $[0,1] \times[0, a]$. Periodic functions are pulled back to functions satisfying (2.19).

Let us normalize $\Xi_{0}$ to have norm one,

$$
\Xi_{0}=\frac{1}{\sqrt{n^{2} A^{2}+m^{2} B^{2}}}(n A, m B), \quad \Xi_{0}^{\perp}=\frac{1}{\sqrt{n^{2} A^{2}+m^{2} B^{2}}}(-m B, n A) .
$$

The change of coordinates in $\mathbb{R}^{2}$,

$$
F:(x, y) \longmapsto z=F(x, y)=x \Xi_{0}^{\perp}+y \Xi_{0},
$$

is orthogonal and hence $-\Delta_{z}=D_{x}^{2}+D_{y}^{2}$.

We have the following simple lemma:

Lemma 2.2. Suppose that $\Xi_{0}$ and $F$ are given by (2.17) and (2.18). If $u=u(z)$ is perodic with respect to $A \mathbb{Z} \times B \mathbb{Z}$ then

$$
F^{*} u(x+k a, y+\ell b)=F^{*} u(x, y-k \gamma), \quad k, \ell \in \mathbb{Z}, \quad(x, y) \in \mathbb{R}^{2},
$$

where, for any fixed $p, q \in \mathbb{Z}$,

$$
a=\frac{(q n-p m) A B}{\sqrt{n^{2} A^{2}+m^{2} B^{2}}}, \quad b=\sqrt{n^{2} A^{2}+m^{2} B^{2}}, \quad \gamma=-\frac{p n A^{2}+q m B^{2}}{\sqrt{n^{2} A^{2}+m^{2} B^{2}}} .
$$

When $B / A=r / s \in \mathbb{Q}, r, s \in \mathbb{Z} \backslash\{0\}$, then

$$
F^{*}(x+k \widetilde{a}, y+\ell b)=F^{*} u(x, y), \quad k, \ell \in \mathbb{Z}, \quad(x, y) \in \mathbb{R}^{2},
$$

for $\widetilde{a}=\left(n^{2} s^{2}+m^{2} r^{2}\right) a$.

Proof. The proof is a calculation: we need to find $a, b$, and $\gamma$ so that for any $k, \ell \in \mathbb{Z}$ there exist $P, Q \in \mathbb{Z}$ so that

$$
k a \Xi_{0}^{\perp}+(\ell b+k \gamma) \Xi_{0}=P A(1,0)+Q B(0,1) .
$$

Taking $b=\sqrt{n^{2} A^{2}+m^{2} B^{2}}$, we only need to check that this relation holds with $k=1$ and $\ell=0$ :

$$
a \Xi_{0}^{\perp}+\gamma \Xi_{0}=p A(1,0)+q B(0,1),
$$


which can be solved for $a$ and $\gamma$ for any $p$ and $q$. By taking inner products with $\Xi_{0}$, $\Xi_{0}^{\perp}$ we obtain formulae for $a$ and $\gamma$.

When $B / A=r / s, r, s \in \mathbb{Z} \backslash\{0\}$ we need to find $M \in \mathbb{Z} \backslash\{0\}$, so that $M \gamma=$ $K b$ for some $K \in \mathbb{Z}$. We check that $M=n^{2} s^{2}+r^{2} m^{2}$ works and hence we obtain periodicity.

Remark 2.2. Condition (2.19) for $w=F^{*} u$ is in fact equivalent to periodicity of $u$ with respect to

$$
\mathbb{Z} \vec{v}_{1} \oplus \mathbb{Z} \vec{v}_{2}, \quad \vec{v}_{1}=(n A, m B), \quad \vec{v}_{2}:=(p A, q B) .
$$

That periodicity is of course implied by periodicity with respect to $A \mathbb{Z} \times B \mathbb{Z}$.

Remark 2.3. A natural choice of $p$ and $q$ which excludes the degenerate cases $p=$ $q=0$ and $p=n, q=m$, can be obtained by assuming (without loss of generality) that $n$ and $m$ are relatively prime and then taking $p$ and $q$ satisfying

$$
n q-m p=1
$$

which is possible by Bezout's theorem. This will be the choice we make in what follows.

We can now give a generalized version of Proposition 2.3:

Proposition 2.4. Suppose that $F: \mathbb{R}^{2} \rightarrow \mathbb{R}^{2}$ is given by (2.18) and that $V \in \mathcal{C}^{\infty}\left(\mathbb{R}^{2}\right)$ is periodic with respect to $A \mathbb{Z} \times B \mathbb{Z}$. Let $a, b$ and $\gamma$ be as in (2.19).

Let $\chi \in \mathcal{C}_{\mathrm{c}}^{\infty}\left(\mathbb{R}^{2}\right)$ is equal to 0 near the set $\eta=0$. There exist operators

$$
Q\left(x, y, h D_{y}\right) \in \mathcal{C}^{\infty}(\mathbb{R}) \otimes \Psi^{0}(\mathbb{R}), \quad R\left(x, y, h D_{y}, h D_{x}\right) \in \Psi^{0}\left(\mathbb{R}^{2}\right),
$$

such that $\left(F^{-1}\right)^{*} Q F^{*}$ and $\left(F^{-1}\right)^{*} R F^{*}$ preserve $A \mathbb{Z} \times B \mathbb{Z}$ periodicity, and

$$
\begin{aligned}
(I+ & h Q)\left(D_{y}^{2}+F^{*} V(x, y)\right) \chi\left(h D_{x}, h D_{y}\right) \\
& =\left(D_{y}^{2}+V_{0}(x)\right)(I+h Q) \chi\left(h D_{x}, h D_{y}\right)+h R,
\end{aligned}
$$

where

$$
V_{0}(x):=\frac{1}{b} \int_{0}^{b} F^{*} V(x, y) d y,
$$

satisfies $V_{0}(x+k a)=V_{0}(x), k \in \mathbb{Z}$.

Proof. We proceed as in the proof of Proposition 2.3. We need to solve equation (2.14) but now $q$ has to satisfy the twisted periodicity condition (2.19), which will follow if $q_{F}(z, \eta):=\left(F^{-1}\right)^{*} q(\bullet, \eta)(z)$ is $A \mathbb{Z} \times B \mathbb{Z}$ periodic. Equation $(2.19)$ is then equivalent to

$$
n \eta\left\langle\Xi_{0}, \partial_{x}\right\rangle q_{F}(z, \eta)=V(z)-\left(F^{-1}\right)^{*} V_{0}(z),
$$

on the support of $\chi(\xi, \eta)$. We note that $\left(F^{-1}\right)^{*} V_{0}$ is the average of $V$ over the (closed) orbit of $\left\langle\Xi_{0}, \partial_{z}\right\rangle$. In particular, the average of the right-hand side is 0 .

An equation of this form can be solved on any compact Riemannian manifold: if $X$ is a length one vectorfield with closed integral curves, and $f$ is function integrating (with respect to the length parameter) to 0 along those curves, then there exists $u$, smooth on $M$, satisfying $X u=f$. To see this, we solve the equation on each curve, demanding that $u$ integrates to zero on that curve. That determines $u$ uniquely and 
hence provides a global smooth solution. Note that this is not the solution we took in (2.15). In the notation of (2.15) the current solution corresponds to

$$
\begin{aligned}
q(x, y, \eta) & =\frac{\mathrm{i} \zeta(\eta)}{2 \eta}\left(\int_{0}^{y}\left(V(x, y)-V_{0}(x)\right) d y-q_{0}(x)\right), \\
q_{0}(x) & :=\frac{1}{B} \int_{0}^{B} \int_{0}^{y}\left(V\left(x, y^{\prime}\right)-V_{0}(x)\right) d y^{\prime} d y
\end{aligned}
$$

Finally, we have the corresponding analogue of Corollarry 2.1.

Corollary 2.2. In the notation of Proposition 2.4, there exist operators

$$
W=W\left(x, y, h D_{x}, h D_{y}\right) \in \Psi^{0}\left(\mathbb{R}^{2}\right), \quad R=R\left(x, y, h D_{x}, h D_{y}\right) \in \Psi^{0}\left(\mathbb{R}^{2}\right),
$$

such that $\left(F^{-1}\right)^{*} W F^{*}$ and $\left(F^{-1}\right)^{*} R F^{*}$ preserve $A \mathbb{Z} \times B \mathbb{Z}$ periodicity, and

$$
\begin{gathered}
(I+h Q)\left(D_{x}^{2}+D_{y}^{2}+V(x, y)\right) \chi\left(h D_{x}, h D_{y}\right) \\
=\left(\left(D_{x}^{2}+D_{y}^{2}+V_{0}(x)\right)(I+h Q)+W\right) \chi\left(h D_{x}, h D_{y}\right)+h R, \\
W(x, y, 0, \eta) \equiv 0 .
\end{gathered}
$$

\section{A semiclassical estimate}

The purpose of this section is to prove the main step towards Theorem 1.2, its semiclassically localized version:

Proposition 3.1. Let $\chi \in \mathcal{C}_{\mathrm{c}}^{\infty}(-1,1)$ be equal to 1 near 0 , and define

$$
\Pi_{h, \rho}\left(u_{0}\right):=\chi\left(\frac{h^{2}(-\Delta+V)-1}{\rho}\right) u_{0}, \quad \rho>0 .
$$

Then for any $T>0$ there exists $\rho, C, h_{0}>0$ such that for any $0<h<h_{0}, u_{0}$, we have

$$
\left\|\Pi_{h, \rho} u_{0}\right\|_{L^{2}}^{2} \leq C \int_{0}^{T}\left\|\mathrm{e}^{-\mathrm{i} t(-\Delta+V)} \Pi_{h, \rho} u_{0}\right\|_{L^{2}(\Omega)}^{2} d t .
$$

Proof. We first observe that if the estimate (3.1) is true for some $\rho>0$, then is is true for all $0<\rho^{\prime}<\rho$. As a consequence, if (3.1) were false, there would exist $T>0$ and sequences

$$
\begin{aligned}
& h_{n} \longrightarrow 0, \quad \rho_{n} \longrightarrow 0, \quad u_{0, n}=\Pi_{h_{n}, \rho_{n}}\left(v_{0, n}\right) \in L^{2} \\
& \mathrm{i} \partial_{t} u_{n}(t, z)=(-\Delta+V(z)) u_{n}(t, z), \quad u_{n}(0, z)=u_{0, n}(z),
\end{aligned}
$$

such that

$$
1=\left\|u_{0, n}\right\|_{L^{2}}^{2}, \quad \int_{0}^{T}\left\|u_{n}(t, \bullet)\right\|_{L^{2}(\Omega)}^{2} d t \longrightarrow 0 .
$$

The sequence $\left(u_{n}\right)$ is bounded in $L_{\text {loc }}^{2}\left(\mathbb{R} \times \mathbb{T}^{2}\right)$ and consequently, after possibly extracting a subsequence, there exists a semi-classical defect measure $\mu$ on $\mathbb{R}_{t} \times T^{*}\left(\mathbb{T}_{z}^{2}\right)$ such that for any function $\varphi \in \mathcal{C}_{0}^{0}\left(\mathbb{R}_{t}\right)$ and any $a \in \mathcal{C}_{\mathrm{c}}^{\infty}\left(T^{*} \mathbb{T}_{z}^{2}\right)$, we have

$$
\langle\mu, \varphi(t) a(z, \zeta)\rangle=\lim _{n \rightarrow \infty} \int_{\mathbb{R}_{t} \times \mathbb{T}^{2}} \varphi(t)\left(a\left(z, h_{n} D_{z}\right) u_{n}\right)(t, z) \bar{u}_{n}(t, z) d t d z .
$$


Furthermore, standard $\operatorname{arguments}^{2}$ show that the measure $\mu$ satisfies

$$
\mu\left(\left(t_{0}, t_{1}\right) \times T^{*} \mathbb{T}_{z}^{2}\right)=t_{1}-t_{0}
$$

- The measure $\mu$ on $\mathbb{R}_{t} \times T^{*}\left(\mathbb{T}^{2}\right)$ is supported in the set

$$
\left\{(t, z, \zeta) \in \mathbb{R}_{t} \times \mathbb{T}_{z}^{2} \times \mathbb{R}_{\zeta}^{2} ;|\zeta|=1\right\}
$$

and is invariant under the action of the geodesic flow:

$$
2\left\langle\zeta, \partial_{z}\right\rangle \mu_{\varphi}=0, \quad \mu_{\varphi}(E):=\int_{\mathbb{R} \times E} \varphi(t) d \mu, \quad E \subset T^{*} \mathbb{T}^{2} .
$$

We shall only use that the support of the measure $\mu$ is invariant:

$$
\left(t_{0}, z_{0}, \zeta_{0}\right) \in \operatorname{supp}(\mu) \Longrightarrow\left(t_{0}, z+s \zeta_{0}, \zeta_{0}\right) \in \operatorname{supp}(\mu), \quad \forall s \in \mathbb{R}
$$

- The measure $\mu$ vanishes on $(0, T) \times T^{*} \Omega$.

We are going to show that the measure $\mu$ is identically equal to 0 on $(0, T) \times T^{*} \mathbb{T}^{2}$. This will provide a contradiction with (3.2).

Remark 3.1. In the case of geometric control, as in the work by Lebeau, the vanishing of $\left.\mu\right|_{(0, T)}$ is a direct consequence of the invariance property. Actually, in Lebeau's work, which concerns boundary value problems, the difficult part is to precisely to prove (analogues of) this invariance property. See the appendix for more details.

The $z$-projection of a trajectory associated with a irrational direction $\zeta$ is dense. Consequently, the support of $\left.\mu\right|_{t \in(0, T)}$ contains only points $\left(t, z, \Xi_{0}\right)$ with rational $\Xi_{0}$ :

$$
\mathbb{T}^{2} \simeq[0, A]_{x} \times[0, B]_{y}, \quad \Xi_{0}=\alpha(A / B, n / m), \quad n, m \in \mathbb{Z}, \quad \alpha \in \mathbb{R} \backslash\{0\} .
$$

In fact, that is the condition implying that the trajectory $s \longmapsto z_{0}+s \Xi_{0}$ is closed when projected to $\mathbb{T}^{2}$, for any $z_{0} \in \mathbb{R}^{2}$. Any other trajectory is dense.

Define

$$
M_{\mu}:=\pi_{1}(\operatorname{supp} \mu \cap\{(t, z, \zeta) ; t \in(0, T)\}), \quad \pi_{1}:(t, z, \zeta) \longmapsto \zeta .
$$

The discussion above shows that $M$ contains only rational directions and hence it is countable and closed. This in turn implies that it contains an isolated point, $\Xi_{0}$ (perfect sets cannot be countable).

We now consider the Schrödinger equation on the strip (or rectangle) $R=\mathbb{R}_{x} \times$ $[0, b]_{y}\left(R=[0, a]_{x} \times[0, b]_{y}\right.$, respectively) using the function $F$ given in (2.18). In this coordinate system, $\Xi_{0}=(0,1)$ - see Figure 1 .

Let $\chi\left(h D_{z}\right)$ be a Fourier multiplier with a symbol supported in a neighbourhood of $\Xi_{0}$ containing no other points in the intersection with $(0, T) \times T^{*} \mathbb{T}^{2}$ of the support of $\mu$, and define

$$
\widetilde{u}_{n}=\chi\left(h_{n} D_{z}\right) u_{n}
$$

We denote by $\widetilde{\mu}$, the semiclassical measure of the sequence $\widetilde{u}_{n}$. We clearly have

$$
\widetilde{\mu}=(\chi(\zeta))^{2} \mu
$$

\footnotetext{
${ }^{2}$ See [1] for a review of recent results about measures used for the Schrödinger equation.
} 
and consequently, we know that the $\zeta$-projection, $\pi_{1}$, of the intersection with $(0, T) \times$ $T^{*} \mathbb{T}^{2}$ of the support of the measure $\widetilde{\mu}$ is equal to $\left\{\Xi_{0}\right\}$ :

$$
M_{\widetilde{\mu}}=\left\{\Xi_{0}\right\}=\{(0,1)\},
$$

where we used the coordinates $(x, y)$ in the last identification.

Using Proposition 2.4 (or, in the easier case of rational tori, Proposition 2.3) we define

$$
v_{n}=(1+h Q) \widetilde{u}_{n}
$$

Since the operator $Q$ is bounded on $L^{2}$, the semiclassical defect measures associated to $v_{n}$ and $\widetilde{u}_{n}$ are equal. We now consider the time-dependent Schrödinger equation satisfied by $v_{n}$. With

$$
Q_{n}:=Q\left(x, y, h_{n} D_{y}\right), \quad R_{n}:=R\left(x, y, h_{n} D_{x}, h_{n} D_{y}\right), \quad W_{n}:=W\left(x, y, h_{n} D_{x}, h_{n} D_{y}\right),
$$

given in $(2.22)$ and $\chi_{n}:=\chi\left(h_{n} D_{z}\right)$, we have

$$
\begin{aligned}
\left(\mathrm{i} \partial_{t}+\Delta-V_{0}(x)\right) v_{n} & =\left(I+h_{n} Q_{n}\right)\left(\mathrm{i} \partial_{t}+\Delta-V(x, y)\right) \chi u_{n}-W_{n} \chi u_{n}-h_{n} R_{n} u_{n} \\
& =-W_{n} \chi_{n} u_{n}+[V, \chi] u_{n}+o_{L^{2}}(1) \\
& =-W_{n} \chi_{n} u_{n}+o_{L_{x, y}^{2}}(1)
\end{aligned}
$$

We also recall that according to Corollary 2.1, the symbol of the operator $W$ vanishes in the set

$$
\{x, y, \xi, \eta): \xi=0\} .
$$

Consequently, it vanishes on the intersection with $(0, T) \times T^{*} \mathbb{T}^{2}$ of the support of the defect measure of $\chi_{n} u_{n}=\widetilde{u}_{n}$ which, by construction, is included in the set

$$
\pi_{1}^{-1}\left(M_{\widetilde{\mu}}\right)=\{(t, x, y, \xi, \eta): \xi=0, \eta=1\} .
$$

As a consequence, the semiclassical measure of $W_{n} \chi_{n} u_{n}$ is equal to 0 . This implies that

$$
\left(\mathrm{i} \partial_{t}+\Delta-V_{0}(x)\right) v_{n}=o_{L_{\mathrm{loc}}^{2}((0, T) \times R)}(1) .
$$

In view of Lemma 2.2, see (2.19), we are now in the setting of Proposition 2.2. To apply it let us choose a band domain $\omega=\omega_{x} \times[0, b]_{y}$ where $\omega_{x}$ is a an interval such that any line $\{x\} \times[0, b]_{y}$, encounters the interior of $\pi_{2}^{-1}(\Omega)$, where $\pi_{2}: R \rightarrow \mathbb{T}^{2}-$ see Figure 2.

We know that there exists $\left(t_{0}, z_{0}, \Xi_{0}\right) \in \operatorname{supp}(\mu)$ for some $t_{0} \in(0, T)$.

Since according to $(3.8)$ on $(0, T)$, the family $\left(v_{n}\right)$ is a family of solutions of the free Schrödinger equations up to $o_{L_{\text {loc }}^{2}((0, T) \times R)}(1)$, we can apply Proposition 2.2 to obtain

$$
\begin{aligned}
\left\|v_{n}\right\|_{L^{2}\left(\left(t_{0}-\epsilon, t_{0}+\epsilon\right) \times R\right)}^{2} & \leq\left\|v_{n}\right\|_{L^{\infty}\left(\left(t_{0}-\epsilon, t_{0}+\epsilon\right) ; R\left(\mathbb{T}^{2}\right)\right)}^{2} \\
& \leq C \int_{t_{0}-2 \epsilon}^{t_{0}+2 \epsilon} \int_{\omega}\left|v_{n}(t, x, y)\right|^{2} d x d y+o(1)
\end{aligned}
$$

where $\epsilon>0$ is chosen small enough so that $\left(t_{0}-2 \epsilon, t_{0}+2 \epsilon\right) \Subset(0, T)$. This implies that there exists $t_{0}^{\prime} \in\left(t_{0}-\epsilon, t_{0}+\epsilon\right), z_{0}^{\prime} \in \omega, \Xi_{0}^{\prime}$ such that

$$
\left(t_{0}^{\prime}, z_{0}^{\prime}, \Xi_{0}^{\prime}\right) \in \operatorname{supp}(\widetilde{\mu}) \text {. }
$$




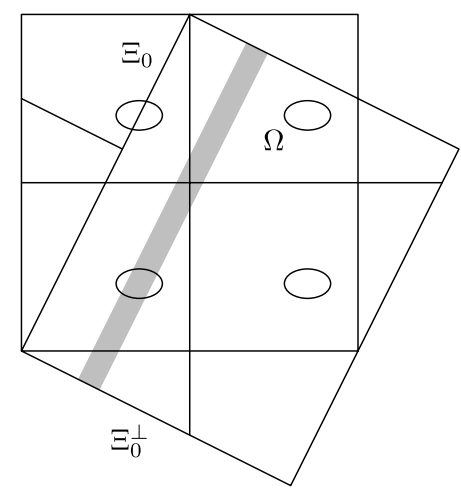

Figure 2. The rectangle $R$, covering a rational torus $\mathbb{T}^{2}$ and the choice of $\omega=\omega_{x} \times[0, b]_{y}$ shown as a shaded region.

From (3.6) we necessarily have $\Xi_{0}^{\prime}=\Xi_{0}$. The invariance of the support of $\widetilde{\mu}$ shows that the whole line

$$
\left(t_{0}^{\prime}, z_{0}^{\prime}+s \Xi_{0}, \Xi_{0}\right) \in \operatorname{supp}(\widetilde{\mu})
$$

consequently the support of the measure $\widetilde{\mu}$ does encounter the set $(0, T) \times T^{*} \Omega$, which gives the contradiction and concludes the proof of Proposition 3.1.

\section{Proof of Theorem 1.2}

To prove Theorem 1.2 we need to pass from the semiclassical estimate of Section 3 to an estimate for all frequencies. We start with a result involving an error term:

Proposition 4.1. For any $T>0$ and any non-empty open set $\Omega \subset \mathbb{T}^{2}$, there exists $C>0$ such that for any $u_{0} \in L^{2}\left(\mathbb{T}^{2}\right)$,

$$
\left\|u_{0}\right\|_{L^{2}}^{2} \leq C\left(\int_{0}^{T} \int_{\Omega}\left|e^{-i t(-\Delta+V)} u_{0}\right|^{2} d z d t+\left\|u_{0}\right\|_{H^{-2}}^{2}\right) .
$$

Remark 4.1. The $H^{-2}$ norm on the right-hand side of (4.1) can be replaced by the $H^{-k}$ norm for any $k$. We only need it for some $k \geq 2$ in order to apply the Bardos-Lebeau-Rauch uniqueness-compactness argument at the end of this section.

Proof. Consider a partition of unity

$$
\begin{gathered}
1=\varphi_{0}(r)^{2}+\sum_{j=1}^{\infty} \varphi_{j}(r)^{2}, \quad \varphi_{j}(r):=\varphi\left(R^{-j}|r|\right), \quad R>1, \\
\varphi \in \mathcal{C}_{\mathrm{c}}^{\infty}\left(\left(R^{-1}, R\right) ;[0,1]\right), \quad\left(R^{-1}, R\right) \subset\{r: \chi(r / \rho) \geq 1 / 2\},
\end{gathered}
$$

where $\chi$ and $\rho$ come from Proposition 3.1. Then, we decompose $u_{0}$ dyadically:

$$
\left\|u_{0}\right\|_{L^{2}}^{2}=\sum_{j=0}^{\infty}\left\|\varphi_{j}\left(P_{V}\right) u_{0}\right\|_{L^{2}}^{2} . \quad P_{V}:=-\Delta+V .
$$


Let $\psi \in \mathcal{C}_{\mathrm{c}}^{\infty}((0, T) ;[0,1])$ satisfy $\psi(t)>1 / 2$, on $T / 3<t<2 T / 3$. We first observe that in Proposition 3.1 we have actually proved (see the remark after Proposition 2.2 ) that

$$
\left\|\Pi_{h} u_{0}\right\|_{L^{2}}^{2} \leq C \int_{\mathbb{R}} \psi(t)^{2}\left\|\mathrm{e}^{-\mathrm{i} t(-\Delta+V)} \Pi_{h} u_{0}\right\|_{L^{2}(\Omega)}^{2} d t, \quad 0<h<h_{0},
$$

which is the version we will use.

Taking $K$ large enough so that $R^{-K} \leq h_{0}$, where $h_{0}$ is as in Proposition 3.1, we apply (4.2) to the dyadic pieces:

$$
\begin{aligned}
\left\|u_{0}\right\|_{L^{2}}^{2} & =\sum_{j}\left\|\varphi_{j}\left(P_{V}\right) u_{0}\right\|_{L^{2}}^{2} \\
& \leq \sum_{j=0}^{K}\left\|\varphi_{j}\left(P_{V}\right) u_{0}\right\|_{L^{2}}^{2}+C \sum_{j=K+1}^{\infty} \int_{0}^{T} \psi(t)^{2}\left\|\varphi_{j}\left(P_{V}\right) \mathrm{e}^{-\mathrm{i} t P_{V}} u_{0}\right\|_{L^{2}(\Omega)}^{2} d t \\
& =\sum_{j=0}^{K}\left\|\varphi_{j}\left(P_{V}\right) u_{0}\right\|_{L^{2}}^{2}+C \sum_{j=K+1}^{\infty} \int_{\mathbb{R}}\left\|\psi(t) \varphi_{j}\left(P_{V}\right) \mathrm{e}^{-\mathrm{i} t P_{V}} u_{0}\right\|_{L^{2}(\Omega)}^{2} d t
\end{aligned}
$$

Using the equation we can replace $\varphi\left(P_{V}\right)$ by $\varphi\left(D_{t}\right)$, which meant that we did not change the domain of $z$ integration. We need to consider the commutator of $\psi \in$ $\mathcal{C}_{\mathrm{c}}^{\infty}((0, T))$ and $\varphi_{j}\left(D_{t}\right)=\varphi\left(R^{-j} D_{t}\right)$. If $\widetilde{\psi} \in \mathcal{C}_{\mathrm{c}}^{\infty}((0, T))$ is equal to 1 on $\operatorname{supp} \psi$ then the semiclassical pseudo-differential calculus with $h=R^{-j}$ (see for instance [12, Chapter 4]) gives

$$
\psi(t) \varphi_{j}\left(D_{t}\right)=\psi(t) \varphi_{j}\left(D_{t}\right) \widetilde{\psi}(t)+E_{j}\left(t, D_{t}\right), \quad \partial^{\alpha} E_{j}=\mathcal{O}\left(\langle t\rangle^{-N}\langle\tau\rangle^{-N} R^{-N j}\right)
$$

for all $N$ and uniformly in $j$.

The errors obtained from $E_{j}$ can be absorbed into the $\left\|u_{0}\right\|_{H^{-2}\left(\mathbb{T}^{2}\right)}$ term on the right-hand side. Hence we obtain

$$
\begin{aligned}
\left\|u_{0}\right\|_{L^{2}}^{2} & \leq C\left\|u_{0}\right\|_{H^{-2}\left(\mathbb{T}^{2}\right)}^{2}+C \sum_{j=0}^{\infty} \int_{0}^{T}\left\|\psi(t) \varphi_{j}\left(D_{t}\right) \mathrm{e}^{-\mathrm{i} t P_{V}} u_{0}\right\|_{L^{2}(\Omega)}^{2} d t \\
& \leq \widetilde{C}\left\|u_{0}\right\|_{H^{-2}\left(\mathbb{T}^{2}\right)}^{2}+C \sum_{j=0}^{\infty}\left\langle\varphi_{j}\left(D_{t}\right)^{2} \widetilde{\psi}(t) \mathrm{e}^{-\mathrm{i} t P_{V}} u_{0}, \widetilde{\psi}(t) \mathrm{e}^{-\mathrm{i} t P_{V}} u_{0},\right\rangle_{L^{2}\left(\mathbb{R}_{t} \times \Omega\right)} \\
& =\widetilde{C}\left\|u_{0}\right\|_{H^{-2}\left(\mathbb{T}^{2}\right)}^{2}+C \int_{\mathbb{R}}\left\|\widetilde{\psi}(t) \mathrm{e}^{-\mathrm{i} t P_{V}} u_{0}\right\|_{L^{2}(\Omega)}^{2} d t \\
& \leq \widetilde{C}\left\|u_{0}\right\|_{H^{-2}\left(\mathbb{T}^{2}\right)}^{2}+C \int_{0}^{T}\left\|\mathrm{e}^{-\mathrm{i} t P_{V}} u_{0}\right\|_{L^{2}(\Omega)}^{2} d t
\end{aligned}
$$

where the last inequality is the statement of the proposition.

To eliminate the $H^{-2}$ error term in (4.1) we use the now classical uniquenesscompactness argument of Bardos et al. [2]. For reader's convenience, we recall the argument.

Let us fix $\delta \geq 0$ and define

$$
N_{\delta}:=\left\{u_{0} \in L^{2}\left(\mathbb{T}^{2}\right): \mathrm{e}^{-\mathrm{i} t(-\Delta+V)} u_{0} \equiv 0 \text { on }(0, T-\delta) \times \Omega\right\} .
$$


Let $u_{0} \in N_{0}$. We now define

$$
v_{\epsilon, 0}=\frac{1}{\epsilon}\left(\mathrm{e}^{-\mathrm{i} \epsilon(-\Delta+V)}-I\right) u_{0} .
$$

If $\epsilon \leq \delta$, then $\mathrm{e}^{-\mathrm{i} t(-\Delta+V)} v_{\epsilon, 0} \equiv 0$ on $(0, T-\delta) \times \Omega$.

We write $u_{0}$ in terms of orthonormal eigenvectors of $-\Delta+V$ :

$$
u_{0}=\sum_{\lambda \in \sigma(-\Delta+V)} u_{0, \lambda} e_{\lambda}
$$

Proposition 4.1 applied with $T$ replaced by $T / 2$ gives that for any $0<\alpha, \beta<T / 2$, we have

$$
\begin{aligned}
\left\|v_{\alpha, 0}-v_{\beta, 0}\right\|_{L^{2}}^{2} & \leq C\left\|v_{\alpha, 0}-v_{\beta, 0}\right\|_{H^{-2}}^{2} \\
& \leq C \sum_{\lambda \in \sigma(-\Delta+V)}\left|\frac{\mathrm{e}^{-\mathrm{i} \alpha \lambda}-1}{\alpha}-\frac{\mathrm{e}^{-\mathrm{i} \beta \lambda}-1}{\beta}\right|^{2}(1+\lambda)^{-2}\left|u_{0, \lambda}\right|^{2} \\
& \leq C^{\prime} \sum_{\lambda \in \sigma(-\Delta+V)} \lambda^{2}|\alpha-\beta|^{2}(1+\lambda)^{-2}\left|u_{0, \lambda}\right|^{2} \leq C^{\prime}|\alpha-\beta|^{2} .
\end{aligned}
$$

Hence, $\lim _{\alpha, \beta \rightarrow 0}\left\|v_{\alpha, 0}-v_{\beta, 0}\right\|_{L^{2}}=0$, and there exists $v_{0} \in L^{2}$ such that

$$
L^{2}-\lim _{\alpha \rightarrow 0} v_{\alpha, 0}=v_{0} \text {. }
$$

This limit is necessarily in $N_{\delta}$ for all $\delta>0$, hence in $N_{0}$. On the other hand, we have in the sense of distributions,

$$
\mathrm{e}^{-\mathrm{i} t(-\Delta+V)} v_{0}=\partial_{t} \mathrm{e}^{-\mathrm{i} t(-\Delta+V)} u_{0}
$$

which implies that

$$
v_{0}=-\mathrm{i}(-\Delta+V) u_{0}
$$

Hence $N_{0}$ is an invariant subspace of $-i(-\Delta+V)$. According to Proposition 4.1, $\left\|u_{0}\right\|_{H^{-2}}$ is a norm on a subspace of $L^{2}, N_{0}$. Hence, the unit ball of $N_{0}$ is compact, and consequently, $N_{0}$ is finite-dimensional. This means that there exists an eigenvector $w$,

$$
(-\Delta+V) w=\mu w,\left.\quad w\right|_{\Omega}=0 .
$$

We can now use the the standard unique continuation results for elliptic second-order operators to conclude that $w \equiv 0$, which then implies that $N_{0}=\{0\}$.

Finally, to conclude the proof of Theorem 1.2, we argue by contradiction: if (1.4) were not true, we could construct a sequence $\left(u_{n, 0}\right) \in L^{2}\left(\mathbb{T}^{2}\right)$ such that

$$
1=\left\|u_{n, 0}\right\|_{L^{2}}, \quad \int_{0}^{T} \int_{\Omega}\left|\mathrm{e}^{-\mathrm{i} t(-\Delta+V)} u_{n, 0}\right|^{2} d x d t \longrightarrow 0, \quad n \longrightarrow \infty .
$$

We could then extract a subsequence $u_{n_{k}, 0}$ converging weakly in $L^{2}$ (and hence strongly in $H^{-2}$ ) to a limit $u_{0} \in N$, which would satisfy, according to Proposition 4.1,

$$
1=\lim _{k \rightarrow \infty}\left\|u_{n_{k}, 0}\right\|_{L^{2}} \leq C \int_{0}^{T} \int_{\Omega}\left|\mathrm{e}^{-\mathrm{i} t(-\Delta+V)} u_{n_{k}, 0}\right|^{2} d x d t+C\left\|u_{n_{k}, 0}\right\|_{H^{-2}}^{2} .
$$

That would imply that

$$
1 \leq C \lim _{k \rightarrow \infty}\left\|u_{n_{k}, 0}\right\|_{H^{-2}}^{2}=C\left\|u_{0}\right\|_{H^{-2}}^{2},
$$


showing that there exists $u_{0} \in N, u_{0} \not \equiv 0$ contradicting our earlier conclusion. This ends the proof of Theorem 1.2.

\section{Acknowledgments}

We would like to thank Semyon Dyatlov, Luc Hillairet, Luc Miller and Claude Zuily for helpful conversations. The first author acknowledges partial support from Agence Nationale de la Recherche project ANR-07-BLAN-0250 and the second author acknowledges partial support by the National Science Foundation under the grant DMS-0654436. He is also grateful to Université de Paris-Nord for its generous hospitality in the Spring 2011 when this paper was written.

\section{Appendix. Proof of Lemma 2.1}

To prove (2.9), we rewrite it as an inequality for periodic functions, that is as an inequality on the circle:

$$
\left\|v_{0}\right\|_{L^{2}\left(\mathbb{T}^{1}\right)}^{2} \leq C \int_{0}^{T}\left\|\mathrm{e}^{\left.-\mathrm{i} t(D+\beta)^{2}+W\right)} v_{0}\right\|_{L}^{2}\left(\omega_{x}\right)^{2} d t
$$

As presented in detail in the second part of Section 4, this follows from the reduction performed in (2.10) and the analogue of estimate (4.1): there exists $C>0$ such that for any $\beta \in[0,2 \pi / a]$, and any $v_{0} \in L^{2}(0, a)$,

$$
\left\|v_{0}\right\|_{L^{2}\left(\mathbb{T}^{1}\right)}^{2} \leq C\left(\int_{0}^{T} \int_{\omega_{x}}\left|\mathrm{e}^{-\mathrm{i} t\left((D+\beta)^{2}+W\right)} v_{0}\right|^{2} d z d t+\left\|v_{0}\right\|_{H^{-2}\left(\mathbb{T}^{1}\right)}^{2}\right) .
$$

We remark that the proof in Section 4 applies to this setting where we consider a family of operators, $\left(D_{x}+\beta\right)^{2}+V, \beta \in[0,2 \pi / a]$, it could actually handle the more general case of a family of potentials $V$, relatively compact in $L^{\infty}$.

As shown in Proposition 4.1 this in turn follows from the analogue Proposition 3.1: for any $T>0$, there exist $C, h_{0}>0$ such that for any $\beta \in[0,2 \pi / a], 0<h<h_{0}$, and $v_{0} \in L^{2}\left(\mathbb{T}^{1}\right)$, we have

$$
\left\|\Pi_{h, \beta} v_{0}\right\|_{L^{2}\left(\mathbb{T}^{1}\right)}^{2} \leq C \int_{0}^{T}\left\|\mathrm{e}^{-\mathrm{i} t\left((D+\beta)^{2}+W\right)} \Pi_{h, \beta} v_{0}\right\|_{L^{2}\left(\omega_{x}\right)}^{2} d t,
$$

where now, in the notation of Proposition 3.1,

$$
\Pi_{h, \beta} v_{0}:=\chi\left(h^{2}\left((D+\beta)^{2}+W\right)-1\right) v_{0}, \quad \beta \in[0,2 \pi / a] .
$$

If these were false there would exist $T>0$ and sequences

$$
\begin{aligned}
& h_{n} \longrightarrow 0, \quad \beta_{n} \longrightarrow \beta \in[0,2 \pi / a], \quad v_{0, n}=\Pi_{h_{n}, \beta_{n}}\left(v_{0, n}\right) \in L^{2}, \\
& \mathrm{i} \partial_{t} v_{n}(t, x)=\left(\left(D+\beta_{n}\right)^{2}+W(x)\right) v_{n}(t, x), \quad v_{n}(0, x)=v_{0, n}(x),
\end{aligned}
$$

such that

$$
1=\left\|v_{0, n}\right\|_{L^{2}\left(\mathbb{T}^{1}\right)}^{2}, \quad \int_{0}^{T}\left\|u_{n}(t, \bullet)\right\|_{L^{2}\left(\omega_{x}\right)}^{2} d t \longrightarrow 0 .
$$

We associate with the sequence $v_{n}$ a semiclassical defect measure, $\nu$, on $\mathbb{R} \times T^{*} \mathbb{T}^{1}$. As recalled in Section 3 (see $[1,20])$ the measure satisfies $\nu\left(\left(t_{0}, t_{1}\right) \times T^{*} \mathbb{T}^{1}\right)=t_{1}-t_{0}$, 
and its support is invariant under the flow of principal symbol of $(D+\beta)^{2}+W(x)$ (since $\left.\beta_{n}=\beta+o(1)\right)$ :

$$
\left(t_{0}, x_{0}, \xi_{0}\right) \in \operatorname{supp}(\nu) \Longrightarrow\left(t_{0}, x_{0}+s \xi_{0}, \xi_{0}\right) \in \operatorname{supp}(\nu), \quad \forall s \in \mathbb{R} .
$$

In view of the second part of (A.4) the measure $\nu$ vanishes on $(0, T) \times T^{*} \omega_{x}$, which contradicts the invariance of the support.

\section{References}

[1] N. Anantharaman and F. Macia, The dynamics of the Schrödinger flow from the point of view of semiclassical measures, arXiv:1102.0970.

[2] C. Bardos, G. Lebeau and J. Rauch, Sharp sufficient conditions for the observation, control, and stabilization of waves from the boundary, SIAM J. Control Optim. 30 (1992), 1024-1065.

[3] N. Burq, Control for Schrodinger equations on product manifolds, unpublished, 1992.

[4] - Contrôle de l'équation des plaques en présence d'obstacles strictement convexes, Mémoire de la S.M.F., 55 (1993), 126 pp.

[5] - Semiclassical estimates for the resolvent in non trapping geometries, Int. Math. Res. Not., 5 (2002), 221-241

[6] N. Burq and P. Gérard, Condition nécessaire et suffisante pour la contrôlabilité exacte des ondes, Comptes Rendus de L'Académie des Sciences, 749-752, t.325, Série I, 1996

[7] N. Burq and G. Lebeau, Mesures de défaut de compacité, application au système de Lamé, Ann. Sci. École Norm. Sup. 4(34) (2001), 817-870.

[8] N. Burq and M. Zworski, Geometric control in the presence of a black box, J. AMS. 17(2) (2004), 443-471.

[9] N. Burq and M. Zworski, Bouncing ball modes and quantum chaos, SIAM Rev., 47 (2005), 43-49.

[10] N. Burq and M. Zworski, Eigenfunctions for partially rectangular billiards, arXiv:math/0312098, unpublished, 2003.

[11] J.-M. Delort, Growth of Sobolev norms of solutions of linear Schrödinger equations on some compact manifolds, Int. Math. Res. Not. IMRN, 11(12) (2010), 2305-2328.

[12] L. C. Evans and M. Zworski, Lectures on semiclassical analysis, http://math.berkeley.edu/ zworski/semiclassical.pdf

[13] P. Gérard and E. Leichtnam, Ergodic properties of eigenfunctions for the Dirichlet problem, Duke Math. J., 71 (1993), 559-607

[14] A. Haraux, Séries lacunaires et contrôle semi-interne des vibrations d'une plaque rectangulaire, J. Math. Pures Appl. 68-4 (1989), 457-465.

[15] A. Hassell, L. Hillairet and J. L. Marzuola, An eigenfunction concentration result for polygonal billiards Comm. P.D.E. 34 (2009), 475-485.

[16] S. Jaffard, Contrôle interne exact des vibrations d'une plaque rectangulaire, Portugal. Math. 47(4) (1990), 423-429.

[17] J. P. Kahane, Pseudo-périodicité et séries de Fourier lacunaires, Ann. Sci. École Norm. Sup. $\mathbf{7 9}(3)$ (1962), 93-150.

[18] G. Lebeau, Contrôle de l'équation de Schrödinger, J. Math. Pures Appl. (9) 71(3) (1992), $267-291$.

[19] J. L. Lions, Contrôlabilité exacte. Perturbation et stabilisation des systèmes distribués, vol. 23 of R.M.A. Masson, 1988.

[20] F. Macià, The Schrödinger flow in a compact manifold: high-frequency dynamics and dispersion, Modern aspects of the theory of partial differential equations, operator theory: advances and applications (216), Springer, Basel, 2011, 275289.

[21] J. L. Marzuola, Eigenfunctions for partially rectangular billiards, Comm. P.D.E. 31 (2006), $775-790$.

[22] L. Miller, Controllability cost of conservative systems: resolvent condition and transmutation J. Funct. Anal. 218(2) (2005), 425-444.

[23] M. Tucsnak and G. Weiss, Observation and Control for Operator Semigroups. Birkhäuser, Basel, 2009. 
[24] A. Weinstein, Asymptotics of eigenvalue clusters for the Laplacian plus a potential, Duke Math. J. 44 (1977), 883-892.

Université Paris Sud, Mathématiques, BÂt 425, 91405, Orsay Cedex, France, And, Ecole Normale Supérieure, 45, rue d'Ulm, 75005 Paris, Cedex 05, France

E-mail address: Nicolas.burq@math.u-psud.fr

Mathematics Department, University of California, Berkeley, CA 94720, USA

E-mail address: zworski@math.berkeley.edu 